\title{
BIORREMEDIACIÓN EFICIENTE DE EFLUENTES METALÚRGICOS MEDIANTE EL USO DE MICROALGAS DE LA AMAZONÍA Y LOS ANDES DEL ECUADOR
}

\author{
Efficient bioremediation of metallurgical effluents through the use of microalgae
} isolated from the Amazonic and highlands of Ecuador

\author{
Nicolás VELA-GARCÍA ${ }^{1}$, María Cristina GUAMÁN-BURNEO ${ }^{2,3}$ y \\ Nory Paola GONZÁLEZ-ROMERO ${ }^{1,2 *}$
}

${ }^{1}$ Facultad de Ingenierías y Ciencias Aplicadas, Ingeniería Ambiental, Sede Queri, calle Queri y Av. de los Granados. Universidad de las Américas, Quito

${ }^{2}$ Laboratorio de Biotecnología Energética, Corporación para la Investigación Energética,. República del Salvador N35-33 y Portugal, Quito

${ }^{3}$ Dirección General de Investigación, Sede Queri, calle Queri y Av. de los Granados, Universidad de las Américas, Quito

*Autora para correspondencia: nory.gonzalezr@gmail.com

(Recibido: febrero 2018; aceptado: noviembre 2018)

Palabras clave: minería, ficorremediación, aurífera, mercurio

\section{RESUMEN}

La explotación minera en el sur del Ecuador ha incrementado su actividad en las últimas décadas debido a la gran riqueza aurífera de la región. No obstante, la extracción de oro involucra procesos que causan impactos perjudiciales al ambiente, tales como la liberación de iones metálicos y compuestos inorgánicos, mismos que son vertidos a sistemas acuáticos o a la superficie terrestre, interactuando de manera adversa con diversos organismos. Debido a esta problemática, el objetivo de esta investigación fue evaluar la eficiencia de remoción de mercurio, fosfatos, sulfatos y nitratos del agua residual provenientes de la actividad minera a través de un proceso de ficorremediación mediante el uso de las microalgas Pleurococcus sp., Chlorella sp. y Scenedesmus sp., cepas aisladas de los Andes y la Amazonía ecuatoriana. Se valoró la adaptación de las microalgas, así como la capacidad de biosorción de los contaminantes del agua residual recolectada del punto de descarga de una de las plantas de beneficio, ubicada a orillas del río Calera, provincia de El Oro, Ecuador. Las microalgas fueron cultivadas para el tratamiento del agua de forma individual y como consorcio en fotobiorreactores. Se caracterizaron los parámetros físicos y químicos del agua residual y del agua tratada, determinándose que todos los tratamientos fueron eficientes, ya que se redujo considerablemente la concentración de contaminantes. El género de microalga con el que mayor porcentaje de remoción de mercurio se obtuvo fue Pleurococcus con el $86 \%$; mientras que, en la remoción de sulfatos, fosfatos, y nitratos, el consorcio de microalgas alcanzó resultados de remoción de contaminantes mayores al $76 \%, 93 \%$ y $97 \%$, respectivamente. Por lo tanto, el uso de microalgas aisladas en los sistemas lacustres de los Andes y la Amazonía del Ecuador representa una fuente de germoplasma promisorio para aplicaciones de biorremediación ambiental.

Key words: mining, phycoremediation, auriferous, mercury 


\begin{abstract}
Mining operations in the south of Ecuador have increased their activity in the last decades due to the great auriferous wealth of the region. Nevertheless, gold extraction involves processes that cause dangerous impacts to the environment, such as the release of metal ions and inorganic compounds, which are discharged into aquatic systems or to the earth's surface, interacting adversely with various organisms. Due to this problem, the aim of this research was to evaluate the removal efficiency of mercury, phosphates, sulfates, and nitrates from the gold mining wastewater through a phytoremediation process. The study collected and applied microalgae species of the Andes and the Ecuadorian Amazon such as Pleurococcus sp., Scenedesmus sp., and Chlorella $\mathrm{sp}$. The adaptation of the microalgae was evaluated, as well as the absorption capacity of the pollutants of the wastewater collected from one of the gold processing plants located on the riverside of the Calera River, province of El Oro. The microalgae strains were cultivated individually and as a consortium in photobioreactors for the treatment. The physical and chemical parameters of the wastewater and the treated water were characterized. The results determined that all the treatments were efficient, since the concentration of contaminants was considerably reduced. Pleurococcus was the genus of microalgae with the highest percentage of mercury removal, with $86 \%$, while in the removal of sulfates, phosphates, and nitrates, the microalgae consortium achieved results greater than $76 \%, 93 \%$ and $97 \%$, respectively. Thus, the use of isolated microalgae on the lacustrine systems of the Andes and the Ecuadorian Amazonia represents a promising source of germplasm for environmental bioremediation applications.
\end{abstract}

\section{INTRODUCCIÓN}

La contaminación del ambiente con metales pesados es una de las principales problemáticas a nivel mundial, ya que se genera por distintos procesos industriales como metalurgia, curtiembre, minería, producción de baterías, galvanizados, etc. (Volesky y Holant 1995, Bwapwa et al. 2017). Este tipo de contaminantes se asocia con un deterioro constante del ambiente, debido a que una vez vertidos en el agua y en la superficie terrestre, los metales pesados presentan el riesgo de volverse tóxicos en altas concentraciones y entrar a la cadena trófica, afectando en gran magnitud la salud del ser humano (WBG 1998, Kumar et al. 2015).

La contaminación por mercurio representa una problemática creciente, especialmente en algunos países de América Latina debido a la expansión de la minería artesanal del oro durante las últimas tres décadas (Appleton et al. 2001). En el Ecuador, la región donde se explota este mineral está ubicada al sur del país, en las provincias de El Oro, Azuay y Zamora Chinchipe, donde los mineros utilizan mercurio para formar una amalgama (INIGEMM 2014).

La producción de oro en 2015 llegó en promedio a 6.2 mil kilogramos, de los cuales el $31.6 \%$ se extrajo de la provincia de El Oro (BCE 2016). Y es en esta zona donde se encuentran las principales plantas de procesamiento o de beneficio de este metal, específicamente, a orillas de los ríos Calera y Amarillo. Incluso, a partir de 2011 se observa un alto incremento del número de plantas de beneficio, de las cuales 85 son consideradas de pequeña producción (Marín 2011, MAE 2013, MME-ARCOMINIGEMM 2016).

Los procesos de extracción de oro generan muchos residuos tóxicos que requieren tecnologías para tratarlos antes de ser emitidos al ambiente. Sin embargo, los métodos tecnológicos que se utilizan en la recuperación de metales pesados conllevan un alto costo de inversión, ya que requieren una alta cantidad de energía, así como reactivos químicos que generan otros desechos igualmente tóxicos (Volesky 2001). Por otro lado, pueden ser ineficientes en la remoción de estos contaminantes, debido a las altas concentraciones que pueden presentarse y su baja eficacia (He y Chen 2014).

Las tecnologías de biorremediación, que son procesos naturales ambientalmente seguros, se han convertido en alternativas atractivas para los métodos convencionales. ellas, la biosorción y la bioacumulación abordan diversas interacciones y concentraciones de metales tóxicos en biomasa viva (bioacumulación) o no viva (bioadsorción) a partir de microorganismos (Bhatia et al. 2016).

Una forma de tratar estos residuos de manera natural y económicamente viable es el uso de microorganismos. Las microalgas tienen la capacidad 
de aprovechar los nutrientes inorgánicos, alta tolerancia a los metales pesados, y habilidad de crecer autotrófica y heterotróficamente, características que benefician su crecimiento (Barsanti y Gualtieri 2006). Las microalgas son microorganismos unicelulares capaces de realizar fotosíntesis, con una gran adaptación ecológica y plasticidad bioquímica (Andersen 2005). Ello las convierte en fuente de varios productos químicos tales como polisacáridos, lípidos, proteínas y pigmentos, entre otros. Estas características han permitido su utilización en diversos campos de la biotecnología durante las últimas tres décadas.

Las microalgas como agentes descontaminantes ofrecen varias ventajas que incluyen bajos costos de producción, fácil manipulación, no son contaminantes y posibilitan la extracción de contaminantes metálicos y sales, por lo que no son considerados una fuente de desechos secundarios (Kumar et al. 2015). Estudios realizados con especies de microalgas solas o en consorcio como Chlorella, Scenedesmus, Nostoc, Cladophora, Chlamydomonas o las cianobacterias Spirulina, Anabaena y Oscillatoria alcanzan altos porcentajes de remoción de contaminantes, en especial para sólidos totales disueltos, $\mathrm{DBO}_{5}, \mathrm{DQO}$, $\mathrm{NO}_{3}, \mathrm{NH}_{4}$ y $\mathrm{PO}_{4}{ }^{3-}$ (Sharma y Khan 2013).

Por este motivo, la ficorremediación es una herramienta con alto potencial de remoción de contaminantes, en la cual las microalgas son usadas como microorganismos depuradores de aguas residuales. Existen algunas especies de microalgas capaces de absorber, acumular y reducir el tiempo de descontaminación de los metales en un ecosistema debido a que la biomasa que se produce a partir de estos microorganismos presenta adaptaciones celulares capaces de retener iones metálicos (Perales-Vela et al. 2006, Kaplan 2013).

El objetivo de este estudio fue evaluar la capacidad de remoción del mercurio, así como la captación de nitratos, fosfatos y sulfatos mediante el uso de microalgas aisladas de áreas protegidas de sistemas lacustres de los Andes y la Amazonía del Ecuador; asimismo, valorar la capacidad de adaptación al efluente metalúrgico entre cepas provenientes de áreas protegidas.

\section{MATERIALES Y MÉTODOS}

\section{Área de estudio}

El presente estudio se realizó con muestras del sobrenadante metalúrgico generado a orillas del río Calera, cantón Portovelo, dentro del distrito minero Zaruma-Portovelo al sur del Ecuador (Fig. 1). El punto de muestreo fue georreferenciado mediante GPS (Garmin ${ }^{\circledR}$ Etrex 30) con las coordenadas $3^{\circ}$ 43' 45" S, 79 38' 11" W. El efluente emitido por las beneficiadoras de oro en el río Calera presentó una temperatura de $23^{\circ} \mathrm{C}$ y $\mathrm{pH}$ de 8 , conductividad de $113.7 \mu \mathrm{S}$, sólidos totales disueltos (STD) de 22.7 ppm y 930 luxes en el área de estudio.

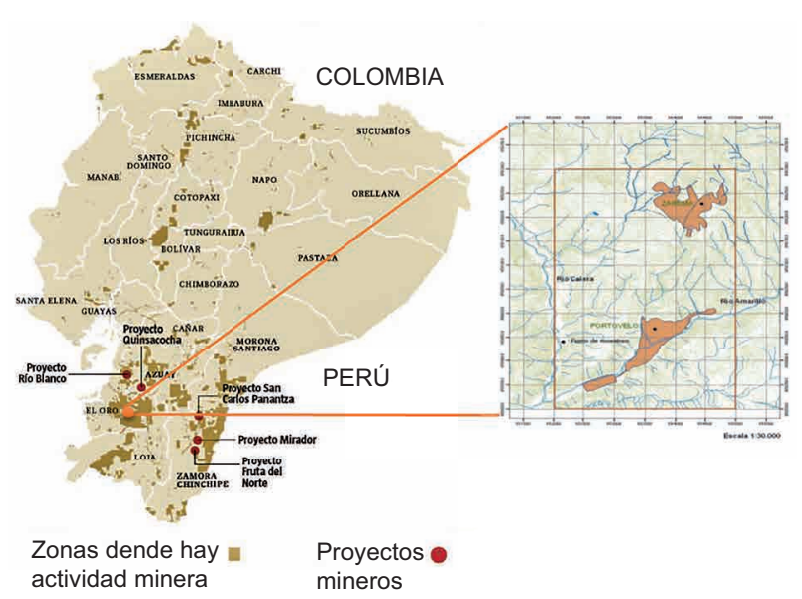

Fig.1. Mapa de las áreas mineras del Ecuador y detalle del sitio de recolección: río Calera, Cantón Portovelo, Provincia de El Oro

La recolección se llevó a cabo a principios de la época seca, cuando el río alcanza un caudal de $9 \mathrm{~m}^{3} / \mathrm{s}$, aproximadamente (Tarras-Wahlberg et al. 2001), con precipitaciones mínimas de $2.1 \mathrm{~m}^{3} / \mathrm{s}$ durante esta época del año y de hasta $20 \mathrm{~m}^{3} / \mathrm{s}$ durante el periodo lluvioso (Tarras-Walhberg y Lane 2003). En la zona de Zaruma-Portovelo, la vegetación es de bosque húmedo templado y la temperatura varía de 14 a $22^{\circ} \mathrm{C}$, con una precipitación media anual de $1500 \mathrm{~mm}$ (GADPEO 2015).

\section{Recolección de muestras de agua}

El muestreo se realizó de acuerdo con la Norma Técnica Ecuatoriana NTE INEN 2169:2013: Agua. Calidad del agua. Muestreo. Manejo y conservación de muestras (INEN 2013). Se emplearon tres envases oscuros de 50, 40 y $30 \mathrm{~L}$. Cada recipiente fue curado con la misma agua residual de donde se extrajo la muestra. Adicionalmente, se tomaron muestras de agua en envases esterilizados de vidrio oscuro de $500 \mathrm{~mL}$ para el análisis de mercurio.

Se midieron los parámetros físicos y químicos del agua recolectada, tales como temperatura, $\mathrm{pH}$, STD y conductividad mediante una sonda multiparamétrica $\left(\mathrm{OAKLON}{ }^{\circledR}\right)$; además, la intensidad de luz 
se midió por medio de un luxómetro (HoldPeak ${ }^{\circledR}$ ) (Guamán-Burneo y González 2016). Asimismo, las muestras fueron acidificadas hasta un $\mathrm{pH}$ inferior a 2 empleando ácido nítrico según la norma establecida. El transporte de las muestras se realizó siguiendo una cadena de frío.

\section{Caracterización física y química del agua residual}

En cada ensayo se realizó un análisis químico del agua residual proveniente del punto de descarga de la beneficiadora de oro, así como un análisis posterior al tratamiento con las microalgas a fin de determinar su capacidad de remoción de mercurio, sulfatos, fosfatos, y nitratos de estos microorganismos, así como su incidencia sobre el $\mathrm{pH}$. El método empleado para el análisis de mercurio se realizó mediante espectrofotometría de absorción atómica de vapor frío APHA 3112 con un rango de detección de 0.0002 a $0.5 \mathrm{mg} / \mathrm{L}$ (APHA 1998).

Para la determinación de sulfatos se utilizó la prueba Nanocolor núm. 0-87 con un rango de detección de 200-1000 mg/L SO ${ }_{4}^{2-}$ y la prueba núm. 0-86 de 10 a $200 \mathrm{mg} / \mathrm{L} \mathrm{SO}_{4}{ }^{2-}$ en una longitud de onda de $436 \mathrm{~nm}$. Se empleó la técnica de enturbiamiento con sulfato de bario mediante el fotómetro MachereyNagel Nanocolor ${ }^{\circledR}$ PF-12.

Se utilizó la prueba Nanocolor núm. 0-80 con un rango de $0.3-15 \mathrm{mg} / \mathrm{L} \mathrm{P}$ con una longitud de onda de $690 \mathrm{~nm}$ para la medición de fosfatos. La determinación fotométrica con azul de molibdeno tras hidrolisis ácida y oxidación a $100-120^{\circ} \mathrm{C}$ se realizó en el fotómetro Macherey-Nagel Nanocolor ${ }^{\mathbb{B}}$ PF-12.

La determinación de nitratos se realizó por medio de la prueba Nanocolor núm. 0-64 con un rango de medida de $0.3-22 \mathrm{mg} / \mathrm{L} \mathrm{NO}_{3}-\mathrm{N}$ en una longitud de onda de $365 \mathrm{~nm}$. La medición se realizó con el fotómetro Macherey-Nagel Nanocolor ${ }^{\mathbb{R}} \mathrm{PF}-12$. El método empleado fue la determinación fotométrica con 2,6-dimetilfenol, en una mezcla de ácido sulfúrico/ácido fosfórico a una temperatura de $20-25^{\circ} \mathrm{C}$ (MN 2009).

\section{Selección e identificación de cepas de microalgas y cianobacterias}

Las cepas de microalgas y cianobacterias fueron recolectadas en diversos sistemas lacustres de áreas protegidas del Ecuador con el Permiso de Investigación núm. 002-14 IC-MC-DNB/MA y el Contrato Marco de Acceso a Recursos Genéticos MAE-DNBCM-2015-0012 otorgado por el Ministerio del Ambiente del Ecuador. Las cepas fueron seleccionadas de acuerdo con la predominancia de especies de cada sistema lacustre estudiado (Cuadro I), las características morfológicas de cada género y la tasa de crecimiento celular en el efluente metalúrgico con un tiempo de experimentación de 30 días.

La identificación se realizó mediante microscopia óptica (SOGERESA ${ }^{\circledR}$ ) utilizando caracteres morfológicos que fueron comparados con claves taxonómicas (Smith 1950, Prescott 1962, Patterson 1996, Wehr et al. 2003, Bellinger y Sigee 2010, Spaulding et al. 2010, Oyadomari 2011, Serediak y Huynh 2011, Baker 2012, Song 2013, Guiry y Guiry 2015, Guamán-Burneo y González 2016).

\section{Determinación de cinética de crecimiento de cepas seleccionadas}

Los medios de cultivo utilizados para el crecimiento microalgal fueron BG-11 (Rippka et al. 1979),

CUADRO I. SELECCIÓN DE CEPAS DE MICROALGAS Y CIANOBACTERIAS DE ACUERDO CON EL CRECIMIENTO CELULAR Y ADAPTACIÓN A EFLUENTES MINEROS

\begin{tabular}{|c|c|c|c|c|}
\hline Código & Género & Sitio de recolección & $\begin{array}{l}\text { Densidad } \\
\text { celular final } \\
(\text { cel } / \mathrm{mL})\end{array}$ & $\begin{array}{l}\text { Tasa de } \\
\text { crecimiento } \\
\text { celular }\end{array}$ \\
\hline CMIE-H4-002 & Scenedesmus sp. & Laguna de Ozogoche, Parque Nacional Sangay & $1.58 \times 10^{7}$ & 0.61 \\
\hline CMIE-D4-001 & Chlorella sp. & Laguna Sucus, Reserva Ecológica Cayambe-Coca & $1.59 \times 10^{7}$ & 0.66 \\
\hline CMIE-B1-001 & Chlorella sp. & Laguna de Muertepungo, Reserva Ecológica Antisana, & $6.50 \times 10^{6}$ & 0.44 \\
\hline CMIE-D5-001 & Chlorella sp. & La Virgen, Reserva Ecológica Cayambe-Coca & $8.41 \times 10^{6}$ & 0.57 \\
\hline CMIE-I8-001 & Pleurococcus sp. & Laguna de Rodeococha, Parque Nacional Llanganates & $1.78 \times 10^{7}$ & 0.70 \\
\hline CMIE-H2-001 & Chlorococcum sp. & Laguna Negra, Parque Nacional Sangay & $7.88 \times 10^{6}$ & 0.51 \\
\hline CMIE-CM-002 & Nostoc sp. & $\begin{array}{l}\text { Lago San Pablo (Donado por la Pontificia Universidad } \\
\text { Católica del Ecuador, Sede Ibarra) }\end{array}$ & $3.93 \times 10^{6}$ & 0.31 \\
\hline Consorcio $\mathrm{C} 1$ & $\begin{array}{l}\text { Chlorella sp. }+ \\
\text { Scenedesmus sp. }\end{array}$ & Reservorio de Cumbayá, Quito & $8.31 \times 10^{6}$ & 0.56 \\
\hline Consorcio CR-002 & $\begin{array}{l}\text { Chlorella } \mathrm{sp.}+ \\
\text { Selenastrum } \mathrm{sp.}\end{array}$ & Reservorio de Cumbayá, Quito & $5.88 \times 10^{6}$ & 0.38 \\
\hline
\end{tabular}


Bold Basal, BBM(Bold 1949, Bischoff y Bold 1963) y Chu núm. 10 (Chu 1942), con el fin de seleccionar el medio óptimo para una mayor tasa de crecimiento de cada cepa y por lo tanto, determinar la curva cinética. El conteo de células vivas se realizó por triplicado en una cámara de Neubauer con una densidad inicial de $1 \times 10^{6} \mathrm{cel} / \mathrm{mL}$. Los cultivos se mantuvieron por un periodo de 30 días en condiciones físicas y químicas de aireación constante, 1500 luxes, de $15-25^{\circ} \mathrm{C}, \mathrm{pH}$ 7 , suministro de $\mathrm{CO}_{2}$ a 5 bares de presión durante 5 minutos tres veces por semana (Guamán-Burneo y González 2016).

\section{Implementación del sistema de tratamiento Ensamblaje de los fotobioreactores}

Para el escalamiento de los cultivos se seleccionaron las cepas que presentaron tolerancia al agua residual con mercurio, sin necesidad de añadir nutrientes para su crecimiento. Se diseñaron cuatro fotobiorreactores para el crecimiento de tres cepas y un consorcio. Cada fotobiorreactor se elaboró con un sistema de iluminación de 1500 luxes, aireación constante, recirculación de agua conectada por una geomembrana, temperatura externa de $15-25^{\circ} \mathrm{C}, \mathrm{pH}$ inicial de 6.9 y un tiempo de retención de 20 días.

Para determinar la cinética de crecimiento de los géneros de microalgas se realizó el conteo celular durante el tiempo de retención, con un inóculo inicial de $1 \times 10^{6} \mathrm{cel} / \mathrm{mL}$.

\section{Determinación de la remoción de mercurio}

El tiempo de remoción de las muestras tratadas con microalgas se realizó mediante la lectura de la absorbancia del mercurio a una longitud de onda de $253.7 \mathrm{~nm}$ (fotómetro Macherey-Nagel Nanocolor ${ }^{\mathbb{B}}$ D500). Los valores referenciales obtenidos antes del tratamiento fueron comparados con las mediciones diarias de los cultivos tratados, los cuales fueron previamente filtrados con mallas de fibra de vidrio. Se calculó el porcentaje de remoción del mercurio en función del tiempo de biosorción de las microalgas, mediante la siguiente fórmula:

$\operatorname{Pr}=\frac{\left(C f_{t} \times 100\right)}{C o_{t}}-100$

donde $\operatorname{Pr}$ es el porcentaje de remoción de mercurio, Co la concentración inicial de un tiempo determinado $t$ y $C f$ la concentración final de un tiempo determinado $t$.

\section{Análisis estadísticos}

Para el análisis estadístico se empleó un diseño completamente aleatorio (DCA) (Gutiérrez y de la
Vara 2008). Las variables evaluadas fueron mercurio, sulfatos, fosfatos y nitratos según su porcentaje de remoción, además de acidificación del agua en cada tratamiento por triplicado de cada fotobiorreactor evaluado. Se realizó la prueba de normalidad de Anderson Darling para evaluar la distribución normal de los resultados a un nivel de significancia de $\mathrm{p} \leq 0.05$.

Adicionalmente, se compararon los tratamientos planteados por un análisis de varianza a un nivel de significancia de $\mathrm{p} \leq 0.0 .5$. Se realizaron pruebas de Levene, comparaciones múltiples (HSD, por sus siglas en inglés) de Tukey y un análisis descriptivo (Sánchez-Otero 2012).

\section{RESULTADOS}

\section{Selección e identificación de cepas de microalgas y cianobacterias \\ Cinética de crecimiento en la etapa de adaptación al mercurio}

De los géneros de microalgas y cianobacterias experimentados en medios de cultivo comerciales, Pleurococcus sp., Chlorella sp. y Scenedesmus sp. fueron los que obtuvieron mayor tasa de crecimiento respecto a las otras especies evaluadas $(0.88,0.86$ y 0.82 generaciones/día, respectivamente) y mayor densidad celular $\left(5.28 \times 10^{7}, 4.92 \times 10^{7}\right.$ y $4.80 \times 10^{7}$ $\mathrm{cel} / \mathrm{mL}$, respectivamente ).

Asimismo, en el agua residual con mercurio, estos géneros de microalgas también obtuvieron la mayor tasa de crecimiento $(0.7,0.66$ y 0.61 generaciones/ día, respectivamente) y mayor densidad celular (1.78 $\times 10^{7}, 1.59 \times 10^{7}$ y $1.58 \times 10^{7} \mathrm{cel} / \mathrm{mL}$, respectivamente). Siendo así, se seleccionaron estas tres cepas $\mathrm{y}$ un consorcio para estudios posteriores.

\section{Implementación del sistema de tratamiento Determinación de la cinética de crecimiento de cepas seleccionadas}

El consorcio microalgal seleccionado (Pleurococcus sp., Scenedesmus sp. y Chlorella sp.) alcanzó la mayor densidad celular de $6.28 \times 10^{7}$ $\mathrm{cel} / \mathrm{mL}$ con una alta tasa de crecimiento celular de 0.94 generaciones/día. Scenedesmus sp. alcanzó $5.79 \times 10^{7} \mathrm{cel} / \mathrm{mL}$ y una tasa de crecimiento de 0.92 generaciones/día; Chlorella sp. obtuvo $5.79 \times$ $10^{7} \mathrm{cel} / \mathrm{mL}$ con una tasa de crecimiento de 0.90 , y Pleurococcus sp. se reprodujo hasta una densidad de $4.93 \times 10^{7} \mathrm{cel} / \mathrm{mL}$ con una tasa de crecimiento de 0.88 generaciones/día.

Pleurococcus sp. obtuvo una densidad celular menor, debido a la capacidad de fijarse en la geomembrana 


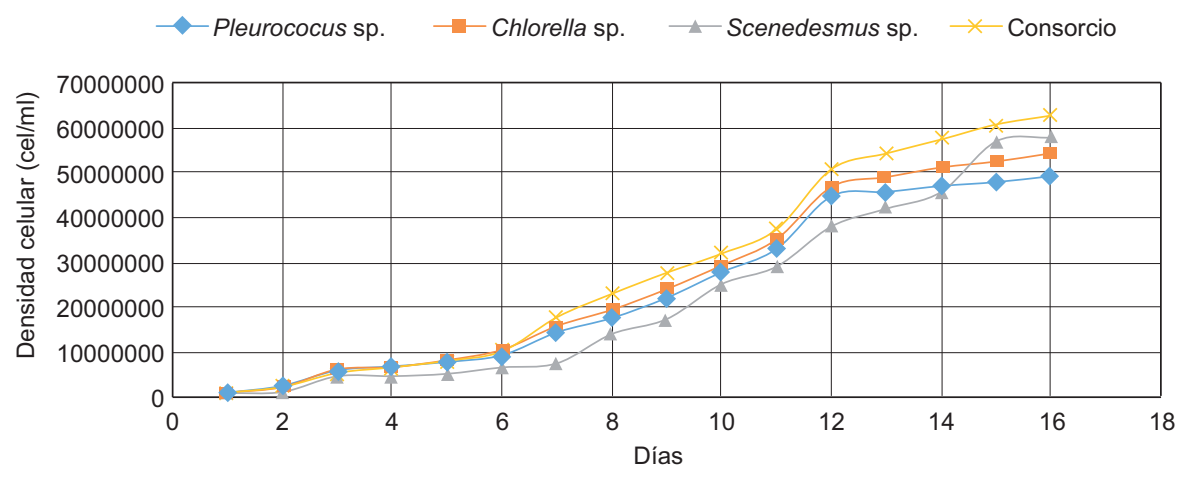

Fig. 2. Cinética de crecimiento de los tres géneros y el consorcio utilizados en la fase de cultivo en los fotobiorractores

formando una biopelícula. Scenedesmus sp. no se fijó a la geomembrana, concentrando todas las microalgas en el agua residual.

En la figura 2 se muestra la curva cinética de crecimiento de cada género de microalga utilizado en la etapa de tratamiento del agua residual en los fotobiorreactores.

\section{Determinación de remoción de mercurio y conta- minantes inorgánicos}

Según los resultados obtenidos para el porcentaje de remoción de $\mathrm{Hg}$ en función del tiempo mediante los análisis estadísticos, existen diferencias altamente significativas $(\mathrm{p} \leq 0.01)$ entre los tratamientos. Como se observa en la figura 3, Scenedesmus sp. demoró en alcanzar su periodo de adaptación e iniciar el proceso de biosorción, removiendo el $64 \%$ de mercurio, mientras que Pleurococcus sp., Chlorella sp. y el consorcio alcanzaron 86.83 y $81 \%$ de remoción de este metal, respectivamente (Cuadro II).

Durante los primeros cinco días de tratamiento con microalgas, se observó que no hubo remoción de Hg. A partir de la segunda semana, Pleurococcus sp., Chlorella sp. y el consorcio empezaron el proceso de biosorción, en tanto que Scenedesmus sp. inició a partir del octavo día. En la figura 4 se indican los tiempos requeridos por los géneros de microalga para remover el mercurio del agua residual.

Los valores de remoción de los contaminantes inorgánicos evaluados se detallan en el cuadro II. Se observaron diferencias altamente significativas ( $\mathrm{p}$ $\leq 0.01$ ) entre los tratamientos para captación de sulfatos, y se determinó que el porcentaje de remoción

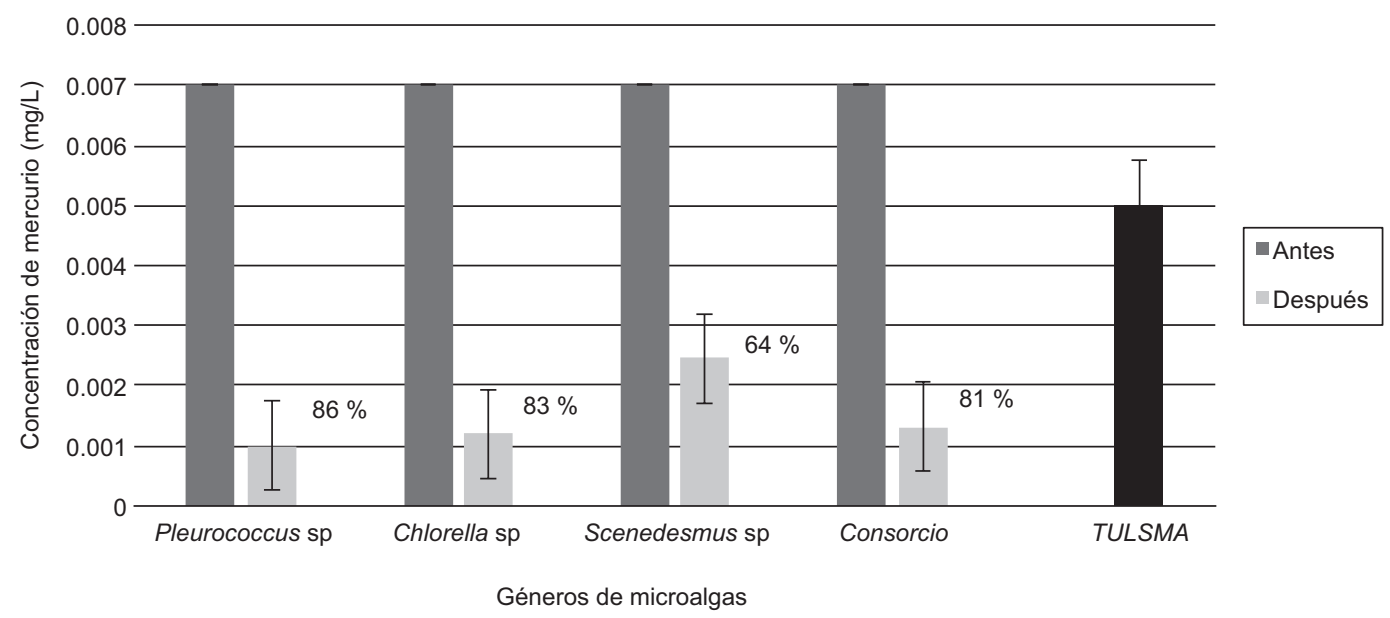

Fig. 3. Concentraciones de mercurio en agua residual antes y después de 20 días de tratamiento en función de la cepa de microalga utilizada en los fotobiorreactores. Se detalla la máxima concentración de mercurio permitida en el Texto Unificado de Legislación Secundaria de Medio Ambiente 
CUADRO II. DISMINUCIÓN DE LA CONCENTRACIÓN DE CONTAMINANTES DELAGUA RESIDUAL EN UN TIEMPO DE REMOCIÓN DE 20 DÍAS

\begin{tabular}{|c|c|c|c|c|c|c|c|c|c|c|}
\hline \multirow{2}{*}{$\begin{array}{l}\text { Género de } \\
\text { microalga }\end{array}$} & \multicolumn{2}{|c|}{ Mercurio (mg/L) } & \multicolumn{2}{|c|}{$\mathrm{pH}$} & \multicolumn{2}{|c|}{ Sulfatos $(\mathrm{mg} / \mathrm{L})$} & \multicolumn{2}{|c|}{ Fosfatos (mg/L) } & \multicolumn{2}{|c|}{ Nitratos $(\mathrm{mg} / \mathrm{L})$} \\
\hline & inicial & final & inicial & final & inicial & final & inicial & final & inicial & Final \\
\hline Pleurococcus sp. & $0.007 \pm 0$ & $0.001 \pm 0$ & & & $1360 \pm 0$ & $453.8 \pm 15$ & $13.8 \pm 0$ & & $44 \pm 0$ & $4.41 \pm 1.2$ \\
\hline Chlorella sp, & $0.007 \pm 0$ & $0.0012 \pm 0$ & $7.0 \pm 0.3$ & $5,36 \pm 0.3$ & $1360 \pm 0$ & $317.13 \pm 15$ & $13.8 \pm 0$ & $2.1 \pm 0.9$ & $44 \pm 0$ & $6.32 \pm 2$ \\
\hline Scenedesmus sp. & $0.007 \pm 0$ & $0.0025 \pm 0$ & $6.9 \pm 0.1$ & $5,50 \pm 0.3$ & $1360 \pm 0$ & $536.35 \pm 25$ & $13.8 \pm 0$ & $2.7 \pm 1$ & $44 \pm 0$ & $7.12 \pm 1.3$ \\
\hline Consorcio & $0.007 \pm 0$ & $0.0013 \pm 0.0001$ & $7.0 \pm 0.3$ & $5,73 \pm 0.2$ & $1360 \pm 0$ & $328.40 \pm 16$ & $13.8 \pm 0$ & $0.4 \pm 0.5$ & $44 \pm 0$ & $2.96 \pm 0.8$ \\
\hline
\end{tabular}

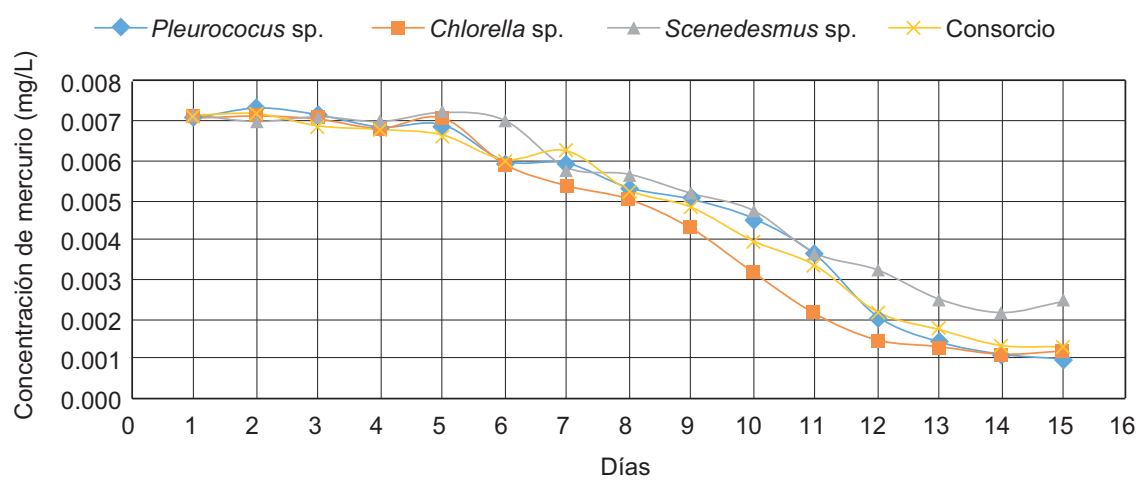

Fig. 4. Disminución de la concentración de mercurio (mg/L) por las microalgas seleccionadas con relación al tiempo

de estas sales difiere entre los géneros utilizados. El género Chlorella sp. fue el que mayor porcentaje de remoción alcanzó $(76.88 \% \pm 1.11 \%)$ en comparación con Pleurococcus sp. y Scenedesmus sp. (60.56 $\pm 1.88 \%$ ).

Según los análisis estadísticos, para la remoción de fosfatos se determinó que no existen diferencias significativas entre tratamientos. El consorcio de microalgas fue el que mayor porcentaje de remoción alcanzó $(97.10 \% \pm 3.81 \%)$. Mientras que en la ficorremediación de nitratos se observaron diferencias significativas $(p \leq 0.05)$ entre los tratamientos, siendo el consorcio el que obtuvo el mayor porcentaje de remoción con $93.12 \% \pm 1.87 \%$.

Para el factor $\mathrm{pH}$ no existieron diferencias significativas, por lo que se determinó que el porcentaje de acidificación es similar en los tratamientos.

\section{DISCUSIÓN}

Caracterización física y química del agua residual

Las aguas del río Calera presentan una alta contaminación, ya que reciben los efluentes ácidos vertidos por las plantas de beneficio en el área de Zaruma-Portovelo, una de las principales zonas mineras del Ecuador.
Al comparar los valores iniciales obtenidos para la concentración de mercurio $(0.007 \mathrm{mg} / \mathrm{L})$ con los establecidos como máximos $(0.005 \mathrm{mg} / \mathrm{L})$ en el Texto Unificado de Legislación Secundaria de Medio Ambiente (TULSMA), libro VI, Norma de calidad ambiental y de descarga de efluentes: Recurso agua (MAE 2015); y con los fijados a nivel internacional por la Association of Metropolitan Sewerage Agencies, cuyo nivel de mercurio permitido para la vida acuática es de $0.0013 \mathrm{mg} / \mathrm{L}$ (AMSA 2002), se observa que las concentraciones de los parámetros analizados en el agua del Río Calera no cumplen con las normativas legales.

Por otro lado, la deforestación en las zonas andinas del Ecuador propician la lixiviación del mercurio natural que se encuentra contenido en los suelos de esta región, lo que acrecentaría el flujo de este metal hacia las zonas bajas de las cuencas hidrográficas (Mainville et al. 2006). De esta manera, tanto el incremento en la actividad minera artesanal como la deforestación, pueden ser responsables del aumento del mercurio (Mora et al. 2016) en los ríos donde se vierten los residuos mineros. Este fenómeno ocurre a nivel mundial, como se detaca en estudios realizados en los ríos que han sido impactados por la minería artesanal en Ghana, donde las concentraciones de mercurio total después de las plantas de beneficio 
presentan valores muy parecidos (0.001-0.01 mg/L) en la época seca (Adjei-Kyereme et al. 2015).

Al considerar las recolecciones efectuadas en este estudio, las cuales se llevaron a cabo a inicios de la época seca, se estima que el caudal del río aún estaba alto, lo que permitió obtener bajas concentraciones de mercurio debido a que la corriente arrastra los compuestos hacia el río Puyango. Al comparar estos valores con estudios anteriores (Cuadro III), se observa una disminución desde los años 1996 al 2001 (Tarras-Wahlberg et al. 2000, 2001, García et al. 2012); sin embargo, en 2010 los valores de mercurio obtenidos por Carling et al. (2013) aumentaron a 0.01 $\mathrm{mg} / \mathrm{L}$. En el presente estudio se registró una disminución a $0.007 \mathrm{mg} / \mathrm{L}$, pero estos valores pueden variar considerablemente a mediados de la época seca.

Las principales formas químicas del mercurio son mercurio metálico o elemental $\left(\mathrm{Hg}^{\mathrm{o}}\right)$, mercurio inorgánico o sales de mercurio $\left(\mathrm{Hg}^{+1}, \mathrm{Hg}^{+2}\right)$ y mercurio orgánico. Todas estas formas son tóxicas, aunque con diferentes vías de absorción, manifestaciones clínicas y respuestas a tratamiento (Valderas et al. 2013). Según la Organización Mundial de la Salud (OMS 2013) se ha determinado que el mercurio elemental y el metilmercurio son tóxicos para el sistema nervioso central y periférico.

Además, por su alta capacidad de bioacumulación y concentración en las cadenas alimenticias, estos compuestos afectan a la fauna de los sistemas acuáticos, como demuestran Tarras-Wahlberg et al. (2001), quienes determinaron la presencia de mercurio en niveles significativamente altos en larvas de moscas y peces en la cuenca del río Puyango.

Debido al alto índice de contaminación ocasionada en sistemas acuáticos y el gran impacto para la salud humana, muchos países han desarrollado políticas y regulaciones con el fin de controlar el contenido de descargas contaminadas por metales pesados y otros residuos provenientes de la minería, de manera rentable y con posibilidad de recuperación de metales (Kumar et al. 2015, Bwapwa et al. 2017). De esta manera, en 2013 el Ministerio del Ambiente del Ecuador creó el Programa de Ordenamiento Ambiental Integral 2013-2016 con el fin de "Restaurar las áreas donde se han identificado y caracterizado pasivos ambientales, desarrollando y promulgando los planes de reparación integral en la cuenca del río Puyango" (MAE 2013). Esta normativa pudo contribuir a la disminución de los niveles de mercurio emitidos al ambiente en los últimos años.

Por otro lado, los valores de $\mathrm{pH}$ determinados fluctúan entre 6.9 y 7.4, cumpliendo con el rango estipulado en el TULSMA, donde se detalla un promedio para descargas de 5 a 9. Los valores de $\mathrm{pH}$ corresponden a flujos ácidos de aguas contaminadas con altos índices de carga de metales pesados, que son generados en las plantas de beneficio por lixiviación de metales asociados al procesamiento de oro y a la oxidación de minerales sulfurados (Pineda 2010). Igualmente, procesos oxidativos de la pirita sólida producen un incremento en las concentraciones de metales y metaloides totales y disueltos, fosfatos, nitratos y sulfatos (INIGEMM 2014). Como resultado, los valores obtenidos en el presente estudio sobrepasaron los de $1000 \mathrm{mg} / \mathrm{L}$ de sulfatos, $10 \mathrm{mg} / \mathrm{L}$ de fosfatos y $10 \mathrm{mg} / \mathrm{L}$ de nitratos permitidos por el TULSMA, siendo de 1360, 13.80 y $44 \mathrm{mg} / \mathrm{L}$, respectivamente.

\section{Eficiencia de los tratamientos \\ Cinética de crecimiento en los fotobioreactores}

Los géneros utilizados en esta investigación (Pleurococcus sp., Chlorella sp. y Scenedesmus sp.) tienden a asimilar y transformar en biomasa de manera eficiente los nutrientes presentes en el agua residual. Al comparar los resultados de densidad celular en la etapa de tratamiento del agua residual en los fotobiorreactores y la dominancia del consorcio,

CUADRO III. CONCENTRACIÓN DE Hg TOTAL (mg/L) EN AGUA RESIDUAL PROVENIENTE DE ACTIVIDAD MINERA DE ZARUMA-PORTOVELO, ECUADOR

\begin{tabular}{lccl}
\hline \multirow{2}{*}{ Año de recolección } & \multicolumn{3}{c}{ Localidad } \\
\cline { 2 - 4 } & Río Caleras & Río Amarillo & Río Puyango \\
\hline $1996-1998$ & $1.1 \times 10^{-3}$ & Tarras-Wahlberg et al. 2000 \\
\hline $1998-1999$ & & $5.7 \times 10^{-4}$ & Tarras-Wahlberg et al. 2001 \\
\hline 2001 & & $2.5 \times 10^{-4}$ & \\
\hline 2010 & 0.01 & $2.2 \times \times 10^{-6}$ & García et al. 2012 \\
\hline 2015 & 0.007 & & Carling et al. 2013 \\
\hline
\end{tabular}


se observa que el género Scenedesmus sp., pese a haber alcanzado una gran densidad celular cultivado de manera individual, no iguala el resultado del consorcio (Fig. 2). Esto se debe principalmente a que el género mencionado tardó más tiempo en adaptarse a las condiciones del medio de cultivo empleado.

Según Arnold (2013), algunos géneros de microalgas requieren de un tiempo de adaptación mayor esperado, el cual se atribuye a que los microorganismos tienden a retrasar su crecimiento hasta asimilar por completo el medio de cultivo, causando una disminución en la producción de biomasa. Sin embargo, Guevara y Zaldumbide (2010) afirman en su investigación que las cepas de microalgas que no crecen en el agua residual no necesariamente carecen de la capacidad de fijar metales, ya que la biomasa muerta también los absorbe, obteniéndose porcentajes de remoción de hasta el $99 \%$ de acuerdo con la concentración. Chacón et al. (2004) manifiestan que esto se debe a que, en consorcios, es importante que los géneros de microalgas tengan características similares en cuanto a la capacidad de adaptación, específicamente con relación a la producción de metalotioneínas, ya que al estar en contacto con el agua contaminada con metales pesados deben adquirir resistencia. Al existir un retraso en el periodo de adaptación es muy probable que los géneros que mejor se acoplan aprovechen anticipadamente los nutrientes presentes en el agua residual.

\section{Remoción de mercurio y contaminantes inorgánicos}

El tratamiento de remoción de mercurio por microalgas alcanzó altos valores de descontaminación. El mayor porcentaje lo obtuvo Pleurococcus sp., con un $86 \%$, seguido por Chlorella sp. con una remoción del $83 \%$. El consorcio microalgal y Scenedesmus sp. absorbieron un 81 y $64 \%$, respectivamente, de este metal (Fig. 4). Posiblemente, la remoción eficaz de este metal se debió a que la concentración inicial de mercurio del agua residual empleada fue menor al límite máximo tolerable de toxicidad al que las microalgas pueden ser sometidas $(1.0 \mathrm{mg} / \mathrm{L})$ (Cordero et al. 2005).

El fenómeno de ficorremediación podría haber actuado de dos maneras: por bioacumulación en células vivas y por biosorción en células muertas o productos de la biomasa. Los mecanismos por los cuales las microalgas remueven metales de soluciones acuíferas incluyen la acumulación extracelular en células vivas, biosorción en la superficie celular y acumulación intracelular por actividad microbiana (Kumar et al. 2015).
Por otro lado, Vílchez (2005) explica que la biopelícula de microalgas contribuye a la remoción de mercurio del agua residual por contacto directo entre los sorbatos (iones metálicos) y el biosorbente (biopelícula de microalgas) de manera repetitiva, aumentando de manera significativa el área de contacto para la captación de metales pesados. La característica de ciertos géneros de microalgas de fijarse a una geomembrana y formar biopelículas se debe principalmente a la excreción de una matriz extracelular adhesiva protectora que permite el asentamiento de las microalgas (Domozych y Domozych 2014), tal como se determinó con Pleurococcus sp. en la remoción de mercurio y otros contaminantes.

Los porcentajes de remoción demuestran que las concentraciones de mercurio en el agua residual tratada en los fotobiorreactores cumplen con la normativa legal vigente en todos los tratamientos. Tanto los géneros de microalgas empleados como el consorcio removieron el contaminante con un valor menor a la norma establecida por el TULSMA $(0.005 \mathrm{mg} / \mathrm{L})$ y la AMSA a nivel internacional $(0.0013 \mathrm{mg} / \mathrm{L})$ (AMSA 2002). El valor obtenido en el presente estudio después del tratamiento con microalgas coincidió exactamente con el de la AMSA (Fig. 4).

Los porcentajes de remoción después del tratamiento con microalgas (Cuadro II) para sulfatos demuestran que las concentraciones en el agua residual tratada en los fotobiorreactores cumplen con la normativa legal vigente. El objetivo principal de los sulfatos en el cultivo de microalgas es conservar la presión osmótica y la proporción de los electrolitos, manteniendo el equilibrio iónico en el proceso de biosorción (McCandles y Craigie 1979). Además, los sulfatos son requeridos por las microalgas como componentes activos en el mecanismo de resistencia en medios de cultivo altamente tóxicos (Mera et al. 2014).

Los fosfatos son fundamentales para la formación de ácidos nucleicos y la transferencia de energía en microorganismos. Además, su deficiencia en el medio de cultivo podría limitar la capacidad de crecimiento de las microalgas y, de esta manera, disminuir la capacidad de remoción de contaminantes de los efluentes metalúrgicos (Martínez 2008). Por este motivo, los altos porcentajes de remoción para fosfatos en el agua residual tratada alcanzaron los niveles establecidos en la normativa legal vigente después de todos los tratamientos con microalgas (Cuadro II).

A pesar de que los géneros de microalgas empleados obtuvieron un porcentaje similar de remoción de fosfatos, el consorcio de microalgas presentó mayor eficiencia. Chacón et al. (2004) alcanzaron un porcen- 
taje de remoción de fosfatos con Chlorella sp. del 80 $\%$, menor al obtenido en esta investigación (97\%).

El porcentaje de remoción de fosfatos del género Scenedesmus sp. fue similar al resultado obtenido por González et al. (1997) en el tratamiento de aguas residuales de la curtiembre, donde se define que al estar en contacto con aguas residuales altamente tóxicas, el género mencionado cambia su metabolismo como mecanismo de resistencia, removiendo una menor cantidad de fosfatos y nutrientes del agua residual (Chacón et al. 2004).

Al comparar el porcentaje de remoción de nitratos con el de fosfatos, se determinó que las microalgas asimilaron mayor cantidad de nitratos. Esto se debe a que los nitratos y nitritos son la fuente secundaria de nutrientes para las microalgas después del $\mathrm{CO}_{2}$ (Dávila 2013). Los porcentajes de remoción de nitratos demuestran que las concentraciones de estas sales inorgánicas en el agua residual tratada estuvieron por debajo de los niveles establecidos por la norma $(10 \mathrm{mg} / \mathrm{L})$ (Cuadro II).

Brennan y Owende (2010) demostraron la eficiencia de estos microorganismos para la asimilación de nitratos debido a que son esenciales en el crecimiento microalgal. El consorcio removió de manera más eficiente este compuesto, seguido por los géneros Pleurococcus sp. y Chlorella sp. con porcentajes del 93, 90 y $86 \%$, respectivamente. Li et al. (2011) corroboraron el alto porcentaje de remoción de nitratos y amonio empleando Pleurococcus sp. y Chlorella sp. en su investigación, donde alcanzaron una remoción del 82.5 al $100 \%$, respectivamente.

El género Scenedesmus sp. empleado en la presente investigación fue el menos eficiente en la remoción de nitratos. Sin embargo, Abdel-Raouf et al. (2012) obtuvieron un resultado del $100 \%$ en la remoción de nitratos y nitritos en el tratamiento de aguas residuales municipales al emplear este mismo género de microalga, por lo que se deduce que la presencia de metales pesados influyó de manera directa en la capacidad de captación de nutrientes como los nitratos.

\section{CONCLUSIONES}

El consorcio de Pleurococcus sp., Chlorella y Scenedesmus sp. removió de manera más eficiente los nitratos, fosfatos y sulfatos de los efluentes metalúrgicos en un 97,93 y $76 \%$, respectivamente.

El tratamiento más eficiente para la remoción de mercurio del agua residual fue Pleurococcus sp., que alcanzó un $86 \%$ en un tiempo de retención de 12 días.
Todos los tratamientos planteados en este estudio alcanzaron un alto porcentaje de remoción de mercurio, fosfatos, sulfatos y nitratos, lo cual permite que los efluentes metalúrgicos tratados cumplan con el límite máximo permisible de la normativa legal vigente para cada uno de los parámetros mencionados.

\section{AGRADECIMIENTOS}

A la Empresa Eléctrica Quito (EEQ) de Ecuador, a través de la Gerencia de Proyectos Especiales, Energía Renovable y Eficiencia Energética por el financiamiento para el desarrollo de este trabajo.

\section{REFERENCIAS}

Abdel-Raouf N., Al-Homaidan A. e Ibraheem I. (2012). Microalgae and wastewater treatment. Saudi J. Biol. Sci. 19 (3), 257-275. DOI: 10.1016/j.sjbs.2012.04.005

Adjei-Kyereme Y., Donkor A.K., Golow A.A., Yeboah P.O. y Pwamang J. (2015). Mercury concentrations in water and sediments in rivers impacted by artisanal gold mining in the Asutifi district, Ghana. Res J. Chem. Environ. Sci. 3 (1), 40-48.

Andersen R.A. (2005). Algal culturing techniques. Academic Press, Boston, EUA, 589 pp.

APHA (1998). Standard methods for the examination of water and wastewater. 20a ed. American Public Health Association, Washington, EUA. 1325 pp.

Appleton J.D., Williams T.M., Orbea H. y Carrasco M. (2001). Fluvial contamination associated with artisanal gold mining in the Ponce Enríquez, Portovelo-Zaruma and Nambija areas, Ecuador. Water Air Soil Poll. 131 (1-4), 19-39.

DOI: 10.1023/a:1011965430757

Arnold M. (2013). Sustainable algal biomass products by cultivation in wastewater flows. VTT Technology 147 , Spoo, Finland, 84 pp.

AMSA (2002). Mercury source control and pollution prevention program evaluation. Association of Metropolitan Sewerage Agencies. Larry Walker Associates. Reporte. California, EUA, 62 pp.

Baker A.L. (2012). Phycokey - an image based key to algae (PS protista), cyanobacteria, and other aquatic objects. University of New Hampshire Center for Freshwater Biology [en línea]. http://cfb.unh.edu/phycokey/phycokey.htm 22/07/2015.

BCE (2016). Reporte de minería. Dirección Nacional de Síntesis Macroeconómica. Publicaciones económicas. Banco Central del Ecuador, Quito, Ecuador, 17 pp. 
Barsanti L. y Gualtieri P. (2006). Algae anatomy, biochemistry, and biotechnology. Taylor and Francis Group, Florida, EUA, 301 pp.

Bhatia M., Satish Babu R., Sonawane S.H., Gogate P.R., Girdhar A., Reddy E.R. y Pola M. (2016). Application of nanoadsorbents for removal of lead from water. Int. J. Environ. Sci. Technol. 14 (5), 1135-1154. DOI: $10.1007 / \mathrm{s} 13762-016-1198-6$

Bellinger E.G. y Sigee D. (2010). Freshwater algae. Identification and use as bioindicators. Wiley-Blackwell, Manchester, Reino Unido, 271 pp.

Bischoff H.W. y Bold H.C. (1963). Phycological studies IV. Some soil algae from enchanted rock and related algal species. University of Texas. Austin, EUA, 95 pp.

Bold H.C. (1949). The morphology of Chlamydomonas chlamydogama, sp. Nov. B. Torrey Bot. Club. 76 (2), 101-108. DOI: $10.2307 / 2482218$

Brennan L. y Owende P. (2010). Biofuels from microalgae - A review of technologies for production, processing, and extractions of biofuels and co-products. Renew. Sust. Energ. Rev. 14 (2), 557-577.

DOI: 10.1016/j.rser.2009.10.009

Bwapwa J.K., Jaiyeola A.T. y Chetty R. (2017). Bioremediation of acid mine drainage using algae strains: A review. S. Afr. J. Chem. Eng. 24, 62-70.

DOI: $10.1016 / j$.sajce.2017.06.005

Carling G.T., Díaz X., Ponce M., Pérez L., Nasimba L., Pazmino E., Rudd A., Merugu S., Fernández D., Gale B. y Johnson W. (2013). Particulate and dissolved trace element concentrations in three southern Ecuador rivers impacted by artisanal gold mining. Water Air Soil Poll. 224 (2), 1-16.

DOI: 10.1007/s11270-012-1415-y

Chacón C., Andrade C., Cárdenas C., Araujo I. y Morales E. (2004). Uso de Chlorella sp. y Scenedesmus sp. en la remoción de nitrógeno, fósforo y DQO de aguas residuales urbanas de Maracaibo, Venezuela. Boletín del Centro de Investigaciones Biológicas. Universidad del Zulia, Venezuela 38 (2), 94-108.

Chu S.P. (1942). The influence of the mineral composition of the medium on the growth of the planktonic algae. Part I. Methods and culture media. J. Ecol. 30 (2), 284-325.

DOI: $10.2307 / 2256574$

Cordero J., Guevara M., Morales E. y Lodeiros C. (2005). Efecto de metales pesados en el crecimiento de la microalga tropical Tetraselmis chuii (Prasinophyaceae). Rev. Bio. Trop. 53 (3-4), 325-330.

Dávila A.G. (2013). Evaluación de las condiciones de cultivo autótrofo de microalgas Chlorella para la producción de biodiésel. Tesis de Licenciatura. Colegio de Ciencias e Ingenierías, Universidad San Francisco de Quito. Quito, Ecuador, 113 pp.
Domozych D. y Domozych C. (2014). Multicellularity in green algae: upsizing in a walled complex. Front. Plant Sci. 5 (649), 1-8. DOI: 10.3389/fpls.2014.00649

García M.E., Betancourt O., Cueva E. y Gimaraes J.R.D. (2012). Mining and seasonal variation of the metals concentrations in the Puyango River basin-Ecuador. J. Environ. Prot. 3 (11), 1542-1550. DOI: $10.4236 /$ jep.2012.311170

GADPEO (2015). Plan de desarrollo y ordenamiento territorial de la Provincia de El Oro 2015-2025. Gobierno Autónomo Descentralizado de la Provincia de El Oro. Machala, Ecuador, 273 pp.

González L., Cañizares S. y Baena S. (1997). Efficiency of ammonia and phosphorus removal from a colombial agroindustrial wastewater by the microalgae Chlorella vulgaris and Scenedesmus dimorphus. Bioresource Technol. 60 (3), 259-262.

Guamán-Burneo M.C. y González N.P. (2016). Catálogo de microalgas y cianobacterias de agua dulce del Ecuador: Biodiversidad de los principales géneros de microalgas y cianobacterias encontradas en sistemas lacustres de áreas protegidas de los Andes y Amazonía del Ecuador. Corporación para la Investigación Energética. Quito, Ecuador, 143 pp.

Guevara M. y Zaldumbide D. (2010). La agonía del Puyango: agua, minería y contaminación. Revista Latinoamericana de Estudios Socioambientales 7, 35-37. DOI: 10.17141/letrasverdes.7.2010.885

Guiry M.D. y Guiry G.M. (2015). AlgaeBase. World-wide electronic publication, National University of Ireland, Galway [en línea] http://www.algaebase.org 24/08/2015.

Gutiérrez H. y de la Vara S.R. (2008). Análisis y diseño de experimentos. $2^{\mathrm{a}}$ ed. McGraw Hill Interamericana, México, D.F., México, 545 pp.

He J. y Chen J.P. (2014). A comprehensive review on biosorption of heavy metals by algal biomass: Materials, performances, chemistry, and modeling simulation tools. Bioresource Technol. 160, 67-78.

DOI: 10.1016/j.biortech.2014.01.068

INEN (2013). Norma Técnica Ecuatoriana NTE INEN 2169:2013 Agua. Calidad del agua. Muestreo. Manejo y conservación de muestras. Instituto Ecuatoriano de Normalización. Registro Oficial. 20 de junio de 2013.

INIGEMM (2014). Tipología de las plantas de beneficio de minerales en el Distrito Minero Zaruma- Portovelo, Provincia de El Oro. Instituto Nacional de Investigación Geológico, Minero, Metalúrgico, Quito, Ecuador, $38 \mathrm{pp}$.

Kaplan D. (2013). Absorption and adsorption of heavy metals by microalgae. En: Handbook of microalgal culture: Applied phycology and biotechnology (Richmond A. y Hu Q., Eds.). 2a ed. John Wiley and Sons, Oxford, pp. 602-611. DOI: 10.1002/9781118567166.ch32 
Kumar S.K., Dahms H.U., Won E.J., Lee J.S. y Shin K.H. (2015). Microalgae- A promising tool for heavy metal remediation. Ecotox. Environ. Safe. 113, 329-352. DOI: 10.1016/j.ecoenv.2014.12.019

Li Y., Chen Y.F., Chen P., Min M., Zhou W., Martínez B., Zhu J. y Ruan R. (2011). Characterization of a microalga Chlorella sp. well adapted to highly concentrated municipal wastewater for nutrient removal and biodiesel production. Bioresource Technol. 102 (8), 5138-5144.

DOI: $10.1016 /$ j.biortech.2011.01.091

MAE (2013). Ordenamiento ambiental integral en la cuenca del Río Puyango. Ministerio del Ambiente del Ecuador, Quito, Ecuador, 96 pp.

MAE (2015). Texto unificado de Legislación Secundaria del Ministerio del Ambiente, Libro VI: de la Calidad Ambiental. Acuerdo Ministerial 097A. Ministerio del Ambiente del Ecuador, Registro Oficial, 4 de mayo.

Mainville N., Webb J., Lucotte M., Davidson R., Betancourt O., Cueva E. y Mergler D. (2006). Decrease of soil fertility and release of mercury following deforestation in the Andean Amazon, Napo River Valley, Ecuador. Sci. Total Environ. 368 (1) 88-98.

DOI: $10.1016 /$ j.scitotenv.2005.09.064

Marín S. (2011). Aporte dinámico de cianuro, mercurio, plomo y arsénico en los cauces naturales de los ríos Calera y Amarillo en el distrito minero Portovelo "Zaruma". Tesis de Licenciatura. Escuela de Ingeniería Civil, Universidad Técnica Particular de Loja, Loja, Ecuador, $127 \mathrm{pp}$.

Martínez L. (2008). Eliminación de $\mathrm{CO}_{2}$ con microalgas autóctonas. Tesis de doctorado. Instituto de Recursos Naturales, Universidad de León, León, España, 213 pp.

McCandles E. y Craigie J. (1979). Sulfated polysaccharides in red and brown algae. Ann. Rev. Plant Physiol. 30, 41-53. DOI: 10.1146/annurev.pp.30.060179.000353

Mera R., Torres E. y Abelde J. (2014). Sulphate, more than a nutrient, protects the microalga Chlamydomonas moewusii from cadmiun toxcity. Aquat, Toxicol. 148, 92-103. DOI: 10.1016/j.aquatox.2013.12.034

MME-ARCOM-INIGEMM (2016). Plan nacional de desarrollo del sector minero. Ministerio de Minería del Ecuador-Agencia de Regulación y Control MineroInstituto Nacional de Investigación Geológico, Minero, Metalúrgico, Quito, Ecuador, 308 pp.

MN (2009). Analyses of drinking, surface, ground, process and wastewater. Nanocolor ${ }^{\circledR}$ 500D. Manual. Macherey-Nagel GmbH\&Co, Düren, Alemania, 40 pp.

Mora A., Jumbo-Flores D., González Merizalde M. y Bermeo-Flores S.A. (2016). Niveles de metales en sedimentos de la cuenca del río Puyango, Ecuador. Rev. Int. Contam. Ambie. 32 (4), 385-397.

DOI: 10.20937/RICA.2016.32.04.02
OMS (2013). El mercurio y la salud. Organización Mundial de la Salud [en línea]. http://www.who.int/ es/news-room/fact-sheets/detail/mercury-and-health 24/08/2017.

Oyadomari J. (2011). Keweenaw: Algae. A collection of freshwater algae (and protozoans) from the Keweenaw Peninsula, MI. Finlandia University, Hancock, MI, Michigan Technological University, Houghton MI [en línea]. http://www.keweenawalgae.mtu.edu/index.htm 28/06/2015.

Patterson D.J. (1996). Free-living freshwater protozoa: A colour guide. Manson Publishing, Londres, Reino Unido, 219 pp.

Perales-Vela H.V., Peña-Castro J.M. y Cañizares-Villanueva R.O. (2006). Heavy metal detoxification in eukaryotic microalgae. Chemosphere 64 (1), 1-10. DOI: 10.1016/j.chemosphere.2005.11.024

Pineda E. (2010). Análisis de la contaminación por cianuro, mercurio, plomo y arsénico en los ríos Calera y Amarillo derivada de la explotación minera. Tesis de Licenciatura. Escuela de Ingeniería Civil, Universidad Técnica Particular de Loja, Loja, Ecuador, 125pp.

Prescott G.W. (1962). Algae of the western Great Lakes area. W.C. Brown, Dubuque, EUA, 348 pp.

Rippka R., Deruelles J., Waterbury J.B., Herdman M. y Stanier R.Y. (1979). Generic assignments, strain histories and properties of pure cultures of cyanobacteria. J. Gen. Microbiol. 111 (1), 1-61. DOI: $10.1099 / 00221287-111-1-1$

Sánchez-Otero J. (2012). Introducción a la estadística no paramétrica y al análisis multivariado. Centro de Publicaciones de la Pontificia Universidad Católica del Ecuador, Quito, Ecuador, 276 pp.

Serediak N. y Huynh M.L. (2011). Algae identification laboratory guide: Accompanying manual to the algae identification field guide. Agriculture and Agri-Food Canadá, Ottawa, 40 pp. [en línea]. http://publications. gc.ca/collections/collection_2011/agr/A125-8-1-2011eng.pdf 24/08/2017

Sharma G.K. y Khan S.A. (2013). Int. J. Environ. Eng. Manage. 4 (6), 573-580.

Smith G.M. (1950). The fresh-water algae of the United States. McGraw-Hill, Nueva York, EUA, 719 pp.

Song L. (2013). Freshwater algae culture collection at the Institute of Hydrobiology, China [en línea]. http:// algae.ihb.ac.cn/English/ 13/06/2015.

Spaulding S.A., Lubinski D.J. y Potapova M. (2010). Diatoms of the United States [en línea]. http://westerndiatoms.colorado.edu 04/08/2015.

Tarras-Wahlberg N.H., Flachier A., Fredriksson S.L., Lane S.N., Lundberg B. y Sangfors O. (2000). Environmental impact of small-scale and artisanal gold mining in southern Ecuador: Implications for the setting of 
environmental standards and for the management of small-scale mining operations. AMBIO 29 (8), 484491. DOI: $10.1579 / 0044-7447-29.8 .484$

Tarras-Wahlberg N.H., Flachier A., Lane S.N. y Sangfors O. (2001). Environmental impacts and metal exposure of aquatic ecosystems in rivers contaminated by small scale gold mining: the Puyango river basin, southern Ecuador. Sci. Total Environ. 278 (1-3), 239-261. DOI: $10.1016 / \mathrm{S} 0048-9697(01) 00655-6$

Tarras-Wahlberg N.H. y Lane S.N. (2003). Suspended sediment yield and metal contamination in a river catchment affected by El Niño events and gold mining activities: the Puyango river basin, southern Ecuador. Hydrol. Process. 17 (15), 3101-3123.

DOI: 10.1002/hyp.1297

Valderas J., Mejías M.E., Riquelme J., Aedo K., Aros S. y Barrera F. (2013). Intoxicación familiar por mercurio elemental: Caso clínico. Revista Chilena de Pediatría 84 (1), $72-79$.
Vílchez R. (2005). Eliminación de metales pesados de aguas subterráneas mediante sistemas de lechos sumergidos: Estudio microbiológico de las biopelículas. Tesis de Doctorado. Instituto del Agua, Universidad de Granada, Granada, España, 219 pp.

Volesky B. y Holant Z. R. (1995). Biosorption of heavy metals. Biotechnol. Prog. 11 (3), 235-250.

DOI: $10.1021 / \mathrm{bp} 00033 \mathrm{a} 001$

Volesky B. (2001). Detoxification of metal-bearing effluents: biosorption for the next century. Hydrometallurgy 59 (2-3), 203-216.

DOI: $10.1016 / \mathrm{s} 0304-386 \mathrm{x}(00) 00160-2$

WBG (1998). Pollution prevention and abatement handbook: Toward cleaner production. The International Bank for Reconstruction and Development, World Bank Group, Washington D.C., EUA, 457 pp.

Wehr J., Sheath R. y Kociolek J.P. (2003). Freshwater algae of North America: Ecology and classification. Academic Press, San Diego, EUA, 918 pp. 